\title{
El sector público en la didáctica de la ciencia en España: CSIC y FECYT
}

\author{
The public sector in the Didactic of Science in Spain: CSIC and FECY
}

Ana Bellón Rodríguez ${ }^{1}$

\section{Resumen}

En España, la Ley 14/2011 de la Ciencia, Tecnología e Innovación establece el mandato de las Administraciones Públicas de fomentar actividades conducentes a la mejora de la cultura científica y tecnológica. Se acomete un estudio exploratorio-descriptivo. Para indagar en su relación con la didáctica de la ciencia se ha accedido a sus estatutos y a sus últimas memorias anuales publicadas. Se ha analizado la labor de dos agentes del sector público institucional en la didáctica de la ciencia educativa. Por una parte, el CSIC, organismo público de investigación, que diseña y ejecuta proyectos de divulgación científica con una amplia variedad temática y múltiples formatos dentro y fuera de los centros de investigación y enseñanza. También la FECYT, fundación del sector público, que promueve una convocatoria anual de ayudas para el fomento de la cultura científica, tecnológica e innovadora y un programa de cultura científica dirigido a jóvenes. Ambos elaboran material didáctico docente para el aula. Por todo ello, constituyen una muestra del compromiso de la Administración Pública por complementar en España la formación reglada en ciencia.

Palabras clave: investigación, alumnado, profesorado, divulgación.

\section{Abstract}

In Spain, Law of Science, Technology and Innovation 14/2011, establishes the command of Public Administrations to promote conducive activities to the scientific and technological culture improvement. An exploratory-descriptive study is undertaken. In order to investigate its relationship with the didactics of science, its statutes and its latest published annual reports have been accessed. The work of two agents of the institutional public sector in the didactics of educational science has been analyzed. On the one hand, the CSIC, a public research organization that designs and executes scientific dissemination projects with a wide variety of topics and multiple formats within and outside the research and teaching centers. Also the FECYT, foundation of the public sector, which promotes an annual call for aid for the promotion of scientific, technological and innovative culture and a program of scientific culture aimed at young people. Both elaborate teaching didactic material for the classroom. For all

1 Doctora en Periodismo, responsable de Comunicación del Consejo Superior de Investigaciones Científicas en Galicia y profesora asociada en la Universidad de Santiago de Compostela. E-mails: ana.bellon@csic.es; ana.bellon@usc.es: http://orcid. org/0000-0002-8779-1110

Recibido: 18/12/2018 Aprobado: 27/06/2019

Bellon Rodriguez, A. (2019). El sector público en la didáctica de la ciencia en España: CSIC y FECYT. Ciencia E Interculturalidad, $24(01), 42-52$. https://doi.org/10.5377/rci.v24i01.8000 
these reasons, they constitute a sign of the commitment of the Public Administration to complement in Spain the regulated education in science.

Keywords: research, students, teachers, dissemination.

\section{Introducción}

En España, la Ley 14/2011, de 1 de junio, de la Ciencia, Tecnología e Innovación incluye entre sus objetivos el impulsar la cultura científica, tecnológica e innovadora en todos los sectores de la sociedad y se reconoce la comunicación y divulgación de la cultura científica, tecnológica e innovadora como consustanciales a la carrera investigadora.

En 2002 la Fundación Española para la Ciencia y la Tecnología (FECYT) inicia la elaboración, con periodicidad bienal, de encuestas de percepción social de la ciencia y tecnología. Entre sus resultados destaca la constatación, edición tras edición, de que los encuestados perciben que el nivel de educación científica y técnica que han recibido es bajo o muy bajo, si bien en 2016 respecto a 2014 se registra una importante estabilidad en la percepción que la ciudadanía tiene de su nivel de formación científico-técnica. En todas las ediciones se han incluido preguntas para conocer el grado de alfabetización científica y tecnológica de los encuestados. En la última edición (FECYT: 2016a) se observa que en general tienen muy claro que la Tierra gira alrededor del Sol $(88,3 \%)$, pero todavía dudan en si comer una fruta modificada genéticamente no influye en los genes de la persona que la come $(78,7 \%)$.

Ante este panorama, se acomete un estudio exploratorio-descriptivo que pone el foco en España y en dos entidades del sector público estatal: la propia FECYT, a la que nos acabamos de referir, que se define por ser una fundación del sector público, y el Consejo Superior de Investigaciones Científicas, el mayor organismo público de investigación en este país.

Se pretende dar respuesta a las siguientes preguntas: ¿Qué iniciativas se están promoviendo en España desde el sector público estatal, tomando como referencia estos agentes, para divulgar Investigación, desarrollo e innovación ( $+\mathrm{D}+\mathrm{i})$ desde edades tempranas? ¿Cuáles son y por qué se caracterizan?

\section{Revisión de literatura}

En la didáctica de la ciencia como objeto de estudio cabe citar, entre otros, los trabajos de investigación presentados por Martín y Osorio (2003), Cajas (2001) o Vázquez y Massero (2008). En los congresos de comunicación social de la ciencia celebrados en España se han presentado diversas iniciativas acerca de la didáctica de la ciencia entre el público no especializado, especialmente durante su etapa formativa. Entre ellos, por ejemplo, figuran los de Ricardo (2017), Frías (2017), García (2017) o Mora (2017). 


\section{Materiales y métodos}

Se acomete un estudio exploratorio-descriptivo para indagar en la apuesta por la didáctica de la ciencia de las dos entidades objeto de estudio: la FECYT y el CSIC. Se accede a sus Estatutos y a sus últimas memorias anuales publicadas para obtener la información correspondiente, sintetizarla, presentarla y analizarla.

Se pretende conocer si apuestan o no por la didáctica de la ciencia. En caso afirmativo, se trata de constatar si se trata de un compromiso institucional firme y cuáles son y por qué se definen las principales iniciativas en este ámbito,

\section{Resultados y discusión}

El Consejo Superior de Investigaciones Científicas, uno de los ocho organismos públicos de investigación de la Administración General del Estado. Éstos son, tal y como se apunta en el artículo 47 de la Ley 14/2011, "creados para la ejecución directa de actividades de investigación científica y técnica, actividades de prestación de servicios tecnológicos y aquellas otras actividades de carácter complementario, necesarias para el adecuado progreso científico y tecnológico de la sociedad".

En calidad de Agencia Estatal, se apunta que su objeto es "el fomento, la coordinación, el desarrollo y la difusión de la investigación científica y técnica, de carácter multidisciplinar" y entre sus funciones se incluye "fomentar la cultura científica en la sociedad y colaborar en la actualización de conocimientos en ciencia y tecnología del profesorado de enseñanzas no universitarias". (En el Real Decreto 1730/2007, de 21 de diciembre).

El CSIC tiene una larga tradición en el fomento de la cultura científica a la sociedad en general y al público escolar en particular. Esta actividad, realizada prácticamente desde los orígenes de la institución por los investigadores de forma espontánea, se vio reforzada en 2004 con la creación de un Área de Cultura Científica con sede en la Organización Central (Madrid), y adquirió un nuevo impulso en 2008 con la creación de una Vicepresidencia Adjunta de Cultura Científica. En la actualidad, a la estructura para el fomento de la cultura científica en la Organización Central se une una red de personal distribuido por todo el territorio nacional. En este sentido, conviene incidir en que el CSIC está presente en las 17 Comunidades Autónomas a través de más de 120 institutos y centros de investigación y cuenta con 14 Delegaciones Institucionales.

Las principales áreas de trabajo del CSIC en el ámbito de la cultura científica y tecnológica abarcan desde el diseño de programas divulgativos específicos con motivo de conmemoraciones científicas a la producción editorial, pasando por la educación y la didáctica de la ciencia y la divulgación en la Red. La financiación para dichas 
actividades procede, fundamentalmente, de la propia institución, convocatorias públicas competitivas y convenios con entidades.

Al margen de las actividades que promueve el CSIC durante eventos como la Semana de la Ciencia o la Noche de los Investigadores o de su participación en ferias científicas, si nos ceñimos a proyectos diseñados para el público escolar y para el profesorado de centros docentes se identifican, entre otros, los siguientes programas:

"El CSIC en la Escuela". En marcha desde el año 2000, fue reconocido en 2017 con el Premio Nacional de Educación. Cuenta con la colaboración de la Fundación BBVA. Su objetivo es colaborar en la actualización del conocimiento del profesorado de enseñanzas no universitarias. Para ello, investigadores y docentes trabajan conjuntamente con el fin de investigar en nuevos métodos de enseñanza de la ciencia desde las primeras etapas de la educación. Entre las acciones que se han puesto en marcha en el marco de este programa figura el "Premio Arquímedes a la Labor Investigadora en el Aula", la revista-serie "El CSIC en la Escuela" o la web kids.csic. Esta iniciativa se ha ido implantando en prácticamente todas las Comunidades Autónomas.

"Ciencia en el Barrio". Se desarrolla en Institutos de Educación Secundaria de distritos madrileños, donde se organizan actividades sobre diversos temas de actualidad científica y tecnológica que incluyen desde talleres experimentales a conferencias o clubes de lectura. Tiene el apoyo de la FECYT.

"Ciudad Ciencia", promovido junto con la Obra Social "la Caixa" cuyo fin es que los habitantes de localidades alejadas de los grandes núcleos urbanos conozcan de primera mano la actualidad científica y tecnológica. Incluye la realización de acciones específicamente dirigidas a estudiantes de Primaria, ESO, Bachillerato y Formación Profesional, que se desarrollan tanto en centros de enseñanza como en otros escenarios.

Una acción dirigida específicamente al alumnado de $4^{\circ}$ de ESO y Bachillerato es "Gymkhana de los Mares y los Océanos", cuyo objetivo es dar a conocer al alumnado de estos cursos el ecosistema marino global y que comprenda su funcionamiento. Cuenta con la colaboración de la Obra Social "la Caixa" y se enmarca en el proyecto "El mar a fondo".

En el ámbito autonómico se localizan varias iniciativas de divulgación científica para el alumnado y el profesorado. En Cataluña figura el programa "El CSIC en el Aula- Cataluña". Su cometido es que los institutos y centros de investigación del CSIC en esta Comunidad Autónoma ofrezcan talleres científico-técnico prácticos en los propios centros de enseñanza y visitas a sus laboratorios. Además, el personal investigador de dichos centros presta apoyo a los trabajos de investigación de Bachillerato. También en esta Comunidad Autónoma el CSIC promueve "El mar a fondo", creado y desarrollado en colaboración entre el Instituto de Ciencias del Mar y Obra Social "la 


\section{EDUCACIÓN}

Caixa" para acercar el conocimiento sobre los mares y océanos al ámbito educativo mediante varias propuestas didácticas.

En Aragón destaca la iniciativa "CSIC en el Aula-Aragón". En el marco de este programa, los centros del CSIC en esta Comunidad Autónoma abren sus puertas para que los estudiantes de los diferentes niveles educativos puedan conocer el trabajo que en ellos se realiza mediante visitas guiadas dirigidas por los propios investigadores. Otra apuesta por la divulgación científica al alumnado es “CSI Aragón”, cuyo fin es fomentar la creatividad y las vocaciones científicas entre jóvenes no universitarios. Para ello, los estudiantes tienen que reproducir la actividad habitual de un equipo de investigación mediante la investigación científica de un crimen. En 2016 ha recibido el Premio a la Divulgación Científica en Aragón de Tercer Milenio.

En Galicia una iniciativa de largo recorrido es "Exper-i-Ciencia CSIC Galicia”, cuyo fin es acercar la ciencia a la comunidad educativa mediante visitas de personal científico-técnico a los propios centros de enseñanza. Las charlas pueden ser solicitadas de forma gratuita por cualquier centro de enseñanza gallego y cubren todos los niveles educativos desde infantil hasta bachillerato, incluyendo ciclos formativos y otras enseñanzas oficiales.

En la Comunidad Valenciana el CSIC está presente a través de 11 centros de investigación. De ellos, ocho participan en el programa "Con Ciencia Sé”, que se desarrolla a través de un ciclo de visitas guiadas a institutos de secundaria y centros de formación profesional en la Comunidad Autónoma. Con ello, se pretende dar a conocer el trabajo del CSIC en la citada Comunidad Autónoma al alumnado de $2^{\circ}$ de Bachillerato y Ciclos Formativos de Grado Superior.

El CSIC, además, dispone de tres grandes espacios de divulgación: el Museo Nacional de Ciencias Naturales (Madrid), el Real Jardín Botánico (Madrid) y el Museo Casa de la Ciencia (Sevilla). En estos espacios se ofrecen a lo largo de todo el año actividades y recursos didácticos para el público escolar, tanto durante el periodo lectivo como no lectivo.

El organismo elabora también materiales didácticos, a través de múltiples formatos, acerca de cuestiones relacionadas con la actualidad científica o conmemoraciones científicas. Se pueden descargar gratuitamente desde la web del CSIC.

\section{La Fundación Española para la Ciencia y la Tecnología}

Es una función del sector público. Su labor se enmarca tanto en lo contemplado en la Ley 50/2002, de 26 de diciembre, de fundaciones, como en la Ley 40/2015, de 1 de octubre, de régimen jurídico del sector público. Se rige por la voluntad de los fundadores reflejada en sus Estatutos; por las disposiciones que, en interpretación y desarrollo 
de los mismos, establezca el Patronato; por lo dispuesto en la ley 50/2002 y por el Real Decreto 1337/2005, de 11 de noviembre, por el que se aprueba el Reglamento de fundaciones de competencia estatal o normas que les sustituyan.

Sus Estatutos incluyen entre sus fines y actividades "fomentar el desarrollo de vocaciones científicas para estimular e incrementar el interés por la ciencia y la tecnología de los jóvenes y la participación de los docentes en nuevas formas de transmisión de los contenidos de la ciencia".

Sus tres principales misiones son 1) Impulsar la ciencia, la tecnología y la innovación 2) Promover su acercamiento a la sociedad y 3) Dar respuesta a las necesidades del Sistema Español de Ciencia, Tecnología e Innovación.

La FECYT apoya, coordina y realiza actividades de comunicación y divulgación para todo tipo de públicos, en colaboración con organizaciones públicas y privadas, con el objetivo de seguir avanzando hacia la integración social del conocimiento científico y tecnológico a través de acciones con las que se pretende fomentar el interés por la ciencia, la tecnología y la innovación, así como aumentar la participación ciudadana. En este sentido, destacan las siguientes acciones:

1) Convocatoria de ayudas para el fomento de la cultura científica, tecnológica $y$ de la innovación

2) Programa de la cultura científica dirigido a jóvenes

En 2007, a iniciativa del Gobierno español, se celebró el Año de la Ciencia. En este contexto, el por aquel entonces Ministerio de Educación y Ciencia encomendó a la FECYT la puesta en marcha de una Convocatoria de ayudas para la realización de actividades de promoción de la cultura científica, que contó con un presupuesto total de siete millones de euros y se ha mantenido en el tiempo. Se divide en tres ejes de actuación:

1) Cultura científica, tecnológica y de la innovación. 1.1. Fomento de la cultura científica, tecnológica y de la innovación 1.2. Estudios sobre cultura científica.

2) Creatividad y vocaciones científicas. 2.1. Fomento de la creatividad y vocaciones científicas. 2.2. Ferias de ciencia.

3) Redes de comunicación y divulgación de la ciencia y la innovación. 3.1. Red de UCC+i. 3.2. Red de Museos de Ciencia y Tecnología.

En el marco de la primera convocatoria se pusieron en marcha las Unidades de Cultura Científica y de la Innovación (UCC+i), que actúan de intermediarias entre las instituciones que las acogen y los ciudadanos con el objetivo principal de promocionar la cultura científica, tecnológica y de la innovación a través de actividades de diversa tipología (FECYT, 2016b). Como resultado de dicha convocatoria, se crearon unas 50 unidades distribuidas en universidades y centros de investigación de toda España. En 


\section{EDUCACIÓN}

las sucesivas convocatorias la FECYT puso al alcance de dichas unidades subvenciones para el desarrollo de sus proyectos.

La propia FECYT (FECYT, 2012) definió los tres requisitos que debía cumplir una UCC para ser considerada como tal y pasar a formar parte del Directorio de UCC + i de la FECYT, coordinadora de la Red: 1) existencia de una organización estable con un responsable con dedicación completa 2) presupuesto que evidencia el compromiso de la entidad con la Unidad y 3) nivel de actividad que dependa de la modalidad a la que pertenezca (comunicación, divulgación, formación o investigación). Recientemente, se ha publicado una obra sobre el origen y la evolución de estas unidades (FECYT, 2016c).

En cuanto al programa de cultura científica dirigido a jóvenes, el objetivo es mejorar el nivel de interés y conocimiento científico de los destinatarios para así poder generar una sociedad informada, participativa y responsable en la toma de decisiones sobre I+D+i. Además de las actividades dirigidas al público más joven, desde FECYT se presta apoyo a los docentes como agentes fundamentales en la educación científica. Dentro del citado programa hay varias iniciativas que se presentan a continuación.

"Campus Científico de Verano", que en 2016 celebró su séptima edición. Se dirige a estudiantes de $4^{\circ}$ de ESO y $1^{\circ}$ de Bachillerato, a los que brinda la oportunidad de entrar en contacto con la labor investigadora que se lleva a cabo en las universidades españolas.

"Campamentos de Verano". En ellos, durante cinco días, la sede de Alcobendas del MUNCYT acoge a participantes con edades comprendidas entre los 3 y los 12 años para que conozcan de cerca del mundo de la ciencia a través de diversas actividades.

La "Gira de Monólogos Científicos" es fruto de la colaboración de la FECYT con la Fundación Telefónica y ha celebrado en 2017 su segunda edición. Se trata de un programa dirigido al alumnado y al profesorado $3^{\circ}$ y $4^{\circ}$ de la ESO. Su objetivo es difundir la ciencia de manera amena y divertida.

"Talleres de Programación", que se dirigen al público con edades comprendidas entre los 8 y los 18 años y "Fin de Científico", que en 2016 celebró su VII edición del Fin de Científico. Ambas tienen lugar en el MUNCYT, de Alcobendas.

FOTCIENCIA, iniciativa con más de una década de recorrido y organizada junto con el CSIC con la colaboración de la Fundación Jesús Serra. Su objetivo es acercar la ciencia a los ciudadanos mediante una visión artística y estética sugerida a través de fotografías científicas.

La FECYT edita anualmente una Unidad Didáctica dirigida a profesores de ESO con el fin de apoyar sus labores docentes: la biodiversidad, la luz, las matemáticas... 


\section{Conclusiones}

En pleno siglo XXI, no hay duda de que el conocimiento científico y tecnológico debe ser compartido. Para ello, es necesaria la implicación del personal investigador que genera dicho conocimiento, la apuesta institucional de las entidades para las que desarrolla su labor y que ambos, personal e institución, dispongan de mediadores entre la ciencia y la sociedad con diferentes perfiles para actuar, con la especialización requerida, en los diferentes ámbitos que permiten hacer comunicación social de la ciencia: desde los medios de comunicación a las actividades divulgativas.

Falk y Dierking (2010) han incidido en que "la mayor parte de la educación científica de una persona se hace fuera de un ambiente formal, la mejor manera de aumentar la comprensión del público en relación a la ciencia está en el restante de su vida". En este sentido, se observa que la divulgación científica que realiza el CSIC dirigida a la sociedad durante su etapa formativa en centros de enseñanza está siendo tanto en ese ambiente formal al que aludenestos autores, es decir, en el propio centro de enseñanza, como fuera del mismo, en los propios centros de investigación.

Cáceres y Ribas (1996) apuntan que: "la comprensión pública de la ciencia se considera actualmente como uno de los valores intrínsecos a las sociedades democráticas". Hoy día está asumida por científicos, educadores y divulgadores la necesidad de hacer llegar y de hacer partícipe a la sociedad de la ciencia y la tecnología que los especialistas van construyendo y desarrollando (Blanco, 2004). Uno de los organismos públicos que está apostando por ello, tal y como se ha puesto de manifiesto en este artículo, es el CSIC, pues tiene entre sus áreas de trabajo en el campo de la divulgación científica la educación y didáctica de la ciencia que se realiza en sus centros e institutos de investigación.

Dado que la investigación del CSIC se define por ser multidisciplinar y multisectorial, cubriendo todos los campos del conocimiento, su labor en el ámbito educativo cuenta con un enorme potencial y con un amplio abanico de posibilidades temáticas. A ello se une también el patrimonio de la institución: desde su extensa red de bibliotecas a los buques oceanográficas pasando por colecciones representativas de flora o fauna, entre otros. El CSIC, por tanto, genera conocimiento científico y tecnológico y lo transmite al público no especializado, en este caso al escolar, a través de iniciativas con las que, entre otros cometidos, completan la oferta formativa reglada de las aulas en materias como Biología, Historia, Matemáticas, ofreciendo al alumnado una información acerca de la investigación que podrá resultarle de utilidad tanto a la hora de elegir y de tomar decisiones acerca de su futuro laboral como a la hora de interpretar y seguir los avances científicos y la actualidad científica que recibirá como un ciudadano no especializado en $\mathrm{I}+\mathrm{D}+\mathrm{i}$. Con sus actividades en el ámbito educativo el CSIC cumple un doble cometido. Por una parte, contribuir a la alfabetización 
científica y al incremento de la cultura científica. Por otra parte, dar a conocer a la entidad y sus investigaciones.

En cuanto a la FECYT, se ha ido convirtiendo durante sus 16 años de existencia en un agente financiador determinante para que entidades públicas y privadas, personas físicas y jurídicas, promuevan en España actividades de divulgación científica. La Convocatoria de Ayudas para el Fomento de Actividades de Cultura Científica e Innovación, si bien ha visto reducida su dotación inicial, ha logrado mantenerse en el tiempo y ha mantenido unos niveles elevados tanto en número de solicitudes presentadas como en el de concedidas. Ello se completa, además, con iniciativas propias que acercan al investigador y su día a día al público escolar.

CSIC y FECYT también han apostado por colaborar con el profesorado en la labor de incentivar al alumnado el gusto por el saber científico y tecnológico y en ofrecerle contenidos divulgativos de calidad con los que completar los contenidos curriculares y los materiales de los tradicionales libros de texto.

\section{Agradecimiento}

Esta publicación obtuvo el financiamiento de: El Fondo de Asistencia Internacional de los Estudiantes y Académicos Noruegos, (SAIH).

\section{Lista de referencias}

Blanco López, A. (2004). Relaciones entre la educación científica y la divulgación científica. Revista Euskera sobre Enseñanza y Divulgación de la Ciencia, 1 (2), 70-86.

Cajas, F. (2001). Alfabetización científica y tecnológica: la transposición didáctica del conocimiento tecnológico. Enseñanza de las Ciencias, 19 (2), 243-254.

Consejo Superior de Investigaciones Científicas (2017). Memoria anual 2016. Disponible en http://www.csic.es/web/guest/memorias

Falk, J.H., y Dierking, I.D. (2010). The 95 per cent solution. American Scientist, 98 (6), 486-493.

Fundación Española para la Ciencia y la Tecnología (2016a). VIII Encuesta de Percepción Social de la Ciencia. FECYT: Madrid.

(2016b). Memoria anual 2016. Disponible en https://www.fecyt.es/es/ publicaciones 
. (2016c). UCC+i: origen y evolución (2007-2014). FECYT: Madrid. Disponible en https://www.fecyt.es/es/publicacion/ucci-origen-y-evolucion-2007-2014

(2012). Libro Blanco de las Unidades de Cultura Científica y de la Innovación. FECYT: Madrid. Disponible en https://www.fecyt.es/es/publicacion/ libro-blanco-de-las-unidades-de-cultura-cientifica-y-de-la-innovacion-ucci

Frías Perles, J.J. (2017). De pequeños genios a grandes científicos. Comunicación oral presentada en el VI Congreso de Comunicación Social de la Ciencia. Actas pendientes de publicación en https://www.uco.es/ccsc2017/

García Lladó, A. (2017). Recerkids, acercando la investigación científica a las aulas de Primaria. Comunicación oral presentada en el VI Congreso de Comunicación Social de la Ciencia. Actas pendientes de publicación en https://www.uco.es/ccsc2017/

Martín Gordillo, M., y Osorio, C. (2003). Educar para participar en ciencia y tecnología. Un proyecto para la difusión de la cultura científica. Revista Iberoamericana de Educación (32), 165-210.

Mora Máquez, M. (2017). Trabajando las ciencias desde la etapa de infantil: el desarrollo del Proyecto "El rincón de los experimentos". Comunicación oral presentada en el VI Congreso de Comunicación Social de la Ciencia. Actas pendientes de publicación en https://www.uco.es/ccsc2017/

Ricardo, E.C. (2017). Diálogo entre la comunicación social de la ciencia y la didáctica de la ciencia. Comunicación oral presentada VI Congreso de Comunicación Social de la Ciencia. Actas pendientes de publicación en https://www.uco.es/ccsc2017/

Vázquez, A., y Manassero, MA. (2008). El declive de las actitudes hacia la ciencia de los estudiantes: un indicador inquietante para la educación científica. Revista Euskera sobre Enseñanza y Divulgación de la Ciencia, 5 (3), 274-292.

Leyes, Órdenes, Decretos, Estatutos

Ley 40/2015, de 1 de octubre, de régimen jurídico del sector público. Boletín Oficial del Estado. Madrid, 2 de octubre de 201, número 236, pp. 89411-89530. Recuperado el 1 de octubre de 2017 desde https://www.boe.es/boe/dias/2015/10/o2/pdfs/ BOE-A-2015-10566.pdf

Ley 14/2011, de 1 de junio, de la ciencia, la tecnología y la innovación. Boletín Oficial del Estado. Madrid, 2 de junio de 2011, número 131, pp. 54387-54455. Recuperado el 1 de octubre de 2017 desde https://www.boe.es/boe/dias/2011/06/o2/pdfs/ BOE-A-2011-9617.pdf 


\section{EDUCACIÓN}

Ley 50/2002, de 26 de diciembre, de Fundaciones. Boletín Oficial del Estado. Madrid, 27 de diciembre de 2002, número 310, pp. 45504-45515. Recuperado el 1 de octubre de 2017 desde https://www.boe.es/boe/dias/2002/12/27/pdfs/A45504-45515.pdf

Ley de 24 de noviembre de 1939 creando el Consejo Superior de Investigaciones Científicas. Boletín Oficial del Estado. Madrid, 28 de noviembre de 1939, número 332, pp. 6668-6671. Recuperado el 1 de octubre de 2017 desde http://www.boe. es/datos/pdfs/BOE/1939/332/Ao6668-o6671.pdf

Real Decreto 1730/2007, de 21 de diciembre, por el que se crea la Agencia Estatal Consejo Superior de Investigaciones Científicas y se aprueba su Estatuto. Boletín Oficial del Estado. Madrid, 14 de enero de 2008, número 12, pp. 2476- 2486. Recuperado el 1 de octubre de 2017 desde https://www.boe.es/boe/dias/2008/o1/14/pdfs/ A02476-02486.pdf

Real Decreto 1337/2005, de 11 de noviembre, por el que se aprueba el Reglamento de fundaciones de competencia estatal. Boletín Oficial del Estado. Madrid, 22 de diciembre de 2005, número 279, pp. 38068 a 38082. Recuperado el 1 de octubre de 2017 desde https://www.boe.es/boe/dias/2005/11/22/pdfs/A38068-38082.pdf

Estatutos de la Fundación Española para la Ciencia y la Tecnología. Aprobados por Acuerdo de Patronato de 30 de junio de 2016 y elevados a escritura pública con número de protocolo 1586 en fecha 1 de marzo de 2017 ante el notario de Madrid. Don José Ortiz Rodríguez. Recuperado el 1 de octubre de 2017 desde https://www.fecyt. es/es/info/normativa-de-aplicacion 